\title{
Deep VUV Scintillators for Detectors Working in Cryogenic Environment
}

\author{
Vladimir Babin, Eduard Feldbach, Marco Kirm, Vladimir N. Makhov, and Sebastian Vielhauer
}

\begin{abstract}
The properties of various inorganic scintillator materials with different origin of their VUV luminescence have been studied under pulsed excitation by XUV (50-1000 eV) synchrotron radiation. A comparison of spectral and timing characteristics of these crystalline VUV emitters has been performed. The processes of energy transfer from the host crystal to the luminescence centers of different nature were analyzed from the features of excitation spectra in the regions of inner-shell absorption.
\end{abstract}

Index Terms-Crossluminescence, excitons, fast VUV luminescence, inorganic scintillators, rare earth ions, synchrotron radiation.

\section{INTRODUCTION}

$\mathbf{T}$ HERE exists a growing demand for VUV inorganic scintillators working at low temperatures. For example, experiments on dark matter detection use liquid xenon and argon scintillators, which emit at 175 and $128 \mathrm{~nm}$, respectively [1]. For the development of detection units working in a cryogenic environment, it is very important to have a compact test set-up using scintillator emission in the VUV range at low temperatures with a constant luminescence yield.

Several inorganic scintillator materials with low-temperature VUV luminescence can be proposed for such a test facility. Crossluminescence (CL) materials based on KF binary compounds $\left(\mathrm{KMgF}_{3}, \mathrm{KYF}_{4}, \mathrm{KLuF}_{4}\right)$ emit fast broadband emission $(\tau \sim 1 \mathrm{~ns})$ in the range of 140-200 nm [2], [3]. The light yield of such scintillators is rather low, which is characteristic for $\mathrm{CL}$. The crystals also emit slow luminescence originating from self-trapped excitions (STE), but at lower energy in the UV region. In $\mathrm{MgO}$ very fast (decay time in picosecond range) and intense VUV emission at $162 \mathrm{~nm}$ is observed due to edge luminescence of excitons, accompanied by other slow emissions in the longer wavelength region [4], [5]. $\mathrm{Al}_{2} \mathrm{O}_{3}$ crystals possess fast $(\sim 7 \mathrm{~ns})$ excitonic luminescence at $166 \mathrm{~nm}$ in addition to other fast and slow emissions in the UV region, originating from $\mathrm{F}^{+}$

Manuscript received June 29, 2007; revised December 11, 2007. This work was supported by the Russian Foundation for Basic Research under Grants 05-02-1730 and 06-02-39027-NNSF, the Estonian Science Foundation under Grant 6538, and the European Community Research Infrastructure Action within the FP6 Program under Contract RII3-CT-2004-506008 (IA-SFS).

B. Babin, E. Feldbach, M. Kirm, and S. Vielhauer are with the Institute of Physics, University of Tartu, 51014 Tartu, Estonia (e-mail: vladimir. babin@ut.ee; eduard.feldbach@ut.ee; marco.kirm@ut.ee; bas@ fi.tartu.ee).

V. N. Makhov is with the Institute of Physics, University of Tartu, 51014 Tartu, Estonia, on leave from the Lebedev Physical Institute, Moscow, Russia (e-mail: makhov@fi.tartu.ee).

Color versions of one or more of the figures in this paper are available online at http://ieeexplore.ieee.org.

Digital Object Identifier 10.1109/TNS.2008.922828 and F centers, respectively [6], [7]. Many wide band gap crystals (mainly fluorides but also some oxides) doped with $\mathrm{Nd}^{3+}$, $\mathrm{Er}^{3+}$, or $\mathrm{Tm}^{3+}$ emit VUV luminescence due to interconfigurational $5 d-4 f$ transitions of the respective ion. This luminescence occurs in the range of 160-200 nm depending on the matrix and on the particular ion (see, e.g., [8], [9]). In most cases, the light yield of such scintillators is also quite low. In addition, $\mathrm{Er}^{3+}$ and $\mathrm{Tm}^{3+}$ doped crystals emit mainly slow VUV luminescence. Our recent studies [10]-[12] have shown that several wide band gap materials containing $\mathrm{Gd}^{3+}$ ions emit VUV luminescence at $\sim 125 \mathrm{~nm}$ due to $5 d-4 f$ transitions in $\mathrm{Gd}^{3+}$ with decay times in the range from $\sim 1$ to $8.5 \mathrm{~ns}$, depending on the matrix. To the best of our knowledge, this is the shortest-wavelength (fast) emission observed from ionic materials.

In the present work, low-temperature luminescence of materials mentioned above was studied under tunable XUV excitation $(50-1000 \mathrm{eV})$ by synchrotron radiation from an undulator source at the DORIS storage ring to investigate the scintillators under realistic conditions.

\section{EXPERIMENTAL DETAILS}

The experiments were carried out using a low temperature luminescence set-up for the VUV spectral region connected to the undulator beam-line BW3 of HASYLAB at DESY [13]. Photons generated by two interchangeable undulators are tunable in the energy range of 30-2000 eV by means of Zeiss SX700 monochromator. Luminescence was detected with a 0.4-m monochromator in Seya-Namioka mounting equipped with a microchannel plate type PMT (Hamamatsu 1645 U-09). The spectral working range $\lambda=110-500 \mathrm{~nm}$ of this device was limited on the short-wavelength side by the transmission of the $\mathrm{MgF}_{2}$ window of the PMT, and on the long-wavelength side by the mechanics of the monochromator. The effective time resolution of the detection system was 300 ps (FWHM), with a 130 ps length of the exciting synchrotron pulses. Discrimination between the fast and slow emission was achieved by measuring signals within "short" and "long" time windows $(\Delta t)$ delayed (by $\delta t$ ) with respect to the excitation pulses of synchrotron radiation. The optical resolution of the analyzing monochromator was typically $2 \mathrm{~nm}$.

The following single crystalline samples were studied in this work under XUV excitation: $\mathrm{KMgF}_{3}, \mathrm{MgO}, \mathrm{Al}_{2} \mathrm{O}_{3}, \mathrm{LiGdF}_{4}$, and $\mathrm{GdF}_{3}$. To ensure clean surfaces, most of the crystalline samples were cleaved prior to mounting onto the sample holder, which was attached to a flow-type liquid helium cryostat. The typical size of the irradiated surface of the samples was $5 \times 5 \mathrm{~mm}^{2}$ and the thickness $\sim 1 \mathrm{~mm}$. 


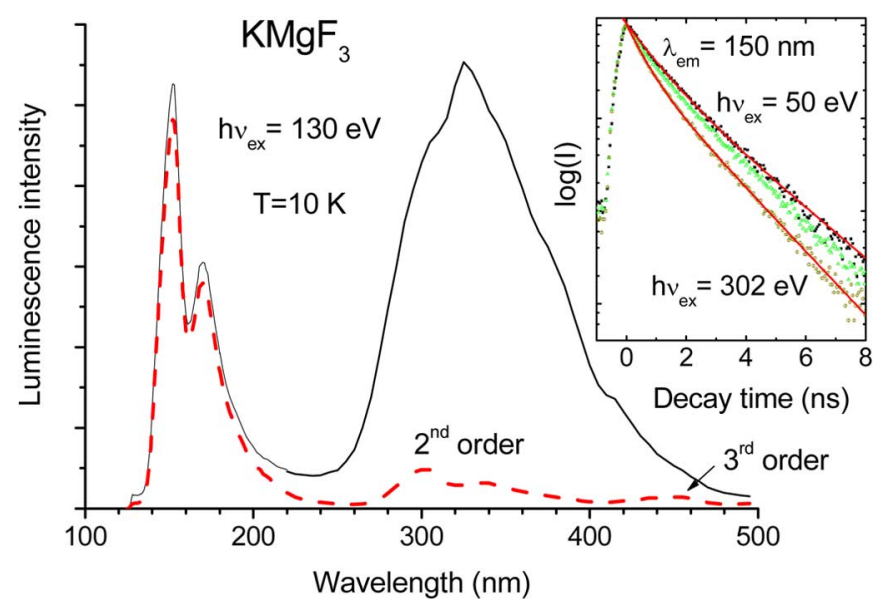

Fig. 1. Fast component (dashed curve, $\Delta t=5.6 \mathrm{~ns}, \delta t=0.15 \mathrm{~ns}$ ) and time-integrated (solid curve) luminescence spectra of $\mathrm{KMgF}_{3}$ measured under excitation by $130 \mathrm{eV}$ photons at a temperature of $10 \mathrm{~K}$. Inset shows decay of fast component (crossluminescence) with gradual shortening of the decay under excitation by photons with increasing energies: 50, 80, and $302 \mathrm{eV}$. Solid lines are the best fits of decay curves by two-exponential decay law for excitation by 50 and $302 \mathrm{eV}$ photons (Log scale is used).

\section{RESULTS AND DISCUSSION}

The luminescence properties and processes of the energy transfer in different VUV-emitting scintillator materials were compared through the analysis of emission and excitation spectra, as well as luminescence decay curves obtained under excitation in the spectral regions corresponding to inner-shell absorption.

In the excitation spectra measured in the XUV region a characteristic decrease of luminescence intensity is usually observed at photon energies corresponding to maxima of absorption. This behavior of the spectra is explained by increased nonradiative near-surface losses of excitations for higher absorption coefficients. Accordingly, excitation and absorption spectra show opposite behavior. In addition, some changes of luminescence decay times are usually observed under variation of excitation energy because of the different quenching mechanisms. However, the influence of these factors strongly depends on the nature of luminescence in a particular crystal and on the mechanisms of the energy transfer from the host crystal to the luminescence centers.

\section{A. Crossluminescence From $\mathrm{KMgF}_{3}$}

$\mathrm{CL}$ is a specific kind of intrinsic luminescence of ionic crystals, which is also called core-valence luminescence, or Augerfree luminescence. It is due to radiative recombination of electrons from the valence band with the holes in the uppermost core band. CL exists in those ionic crystals, in which the Augerdecay of the holes in the uppermost core band is energetically forbidden. CL is observed only from 6 binary ionic crystals $\left(\mathrm{BaF}_{2}, \mathrm{CsF}, \mathrm{CsCl}, \mathrm{CsBr}, \mathrm{RbF}, \mathrm{KF}\right)$, but is also observed from many ternary and multi-component crystals based on combinations of these 6 binary compounds. The shortest-wavelength radiation is emitted by KF-based CL crystals.

Emission spectra of the fast component (CL) and time-integrated luminescence $(\mathrm{CL}+\mathrm{STE})$ obtained from a pure $\mathrm{KMgF}_{3}$ crystal at $10 \mathrm{~K}$ under $130 \mathrm{eV}$ excitation are presented in Fig. 1.

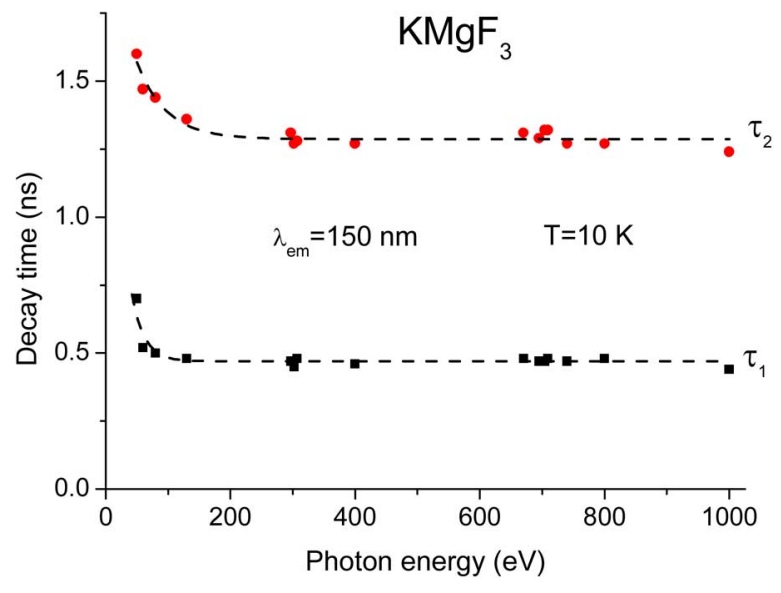

Fig. 2. Decay times of crossluminescence from $\mathrm{KMgF}_{3}$ (at a temperature of $10 \mathrm{~K}$ ) showing shortening of the decay under excitation by photons with increasing energies. Decay curves were fitted by two-exponential decay function. The lines are drawn only for better visualization of data.

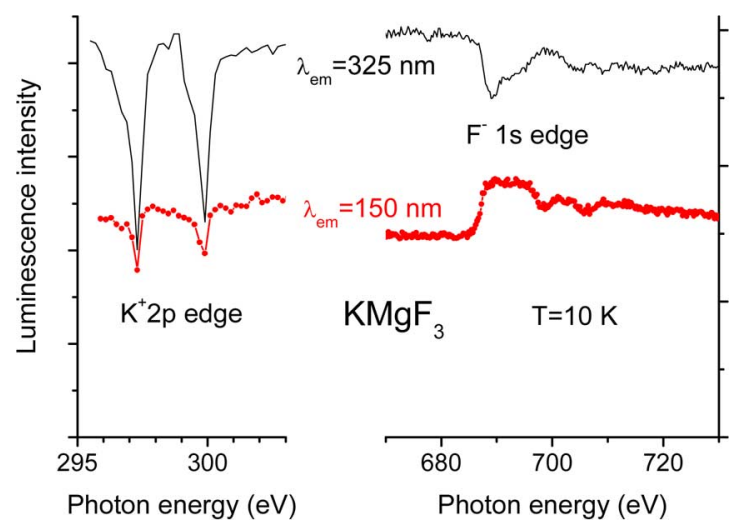

Fig. 3. Excitation spectra of crossluminescence $\left(\lambda_{\mathrm{em}}=150 \mathrm{~nm}\right.$, dotted curves) and self-trapped exciton emission $\left(\lambda_{\mathrm{em}}=325 \mathrm{~nm}\right.$, solid curves $)$ from $\mathrm{KMgF}_{3}$ crystal at $10 \mathrm{~K}$ in the range of the $K 2 p$ and $F 1 s$ absorption. Note the different energy scale for two parts of the Figure.

The CL spectrum consists of two bands centered at 150 and 170 $\mathrm{nm}$. The STE emission band has its maximum at $325 \mathrm{~nm}$. The decay time of CL is of the order of $1 \mathrm{~ns}$, but the decay law is not single-exponential for any of the excitation energies (see Fig. 2). The decay becomes shorter with increasing excitation energy in the range up to $\sim 300 \mathrm{eV}$ and practically does not change for higher excitation energies, including the region above the $F^{-} 1 s$ absorption. The decay curves can be fitted well by a two-exponential decay function with decay components shown in Fig. 2.

Fig. 3 shows the excitation spectra for CL $(150 \mathrm{~nm})$ and STE $\left(325 \mathrm{~nm}\right.$ ) emission in a pure $\mathrm{KMgF}_{3}$ in the energy ranges covering the $\mathrm{K}^{+} 2 p$ and $\mathrm{F}^{-} 1 s$ absorption edges. Maxima in the absorption coefficient cause minima in the excitation spectra at 298.5 and $301 \mathrm{eV}$ corresponding to the doublet of $\mathrm{K}^{+} 2 p_{3 / 2}$ and $2 p_{1 / 2}$ states, in accordance with typical behavior of excitation spectra under inner-shell absorption. However, the depth of minima is larger for STE luminescence. This is due to the much shorter lifetime of excitations for CL $(\sim 1 \mathrm{~ns})$ compared to STE luminescence (at least many $\mu \mathrm{s}$ ). Accordingly, the diffusion length $L=(D \tau)^{1 / 2}$, where $D$ is diffusion coefficient, is much smaller for CL, leading to a weaker influence of near-surface losses on CL compared to STE emission. The intensity of 


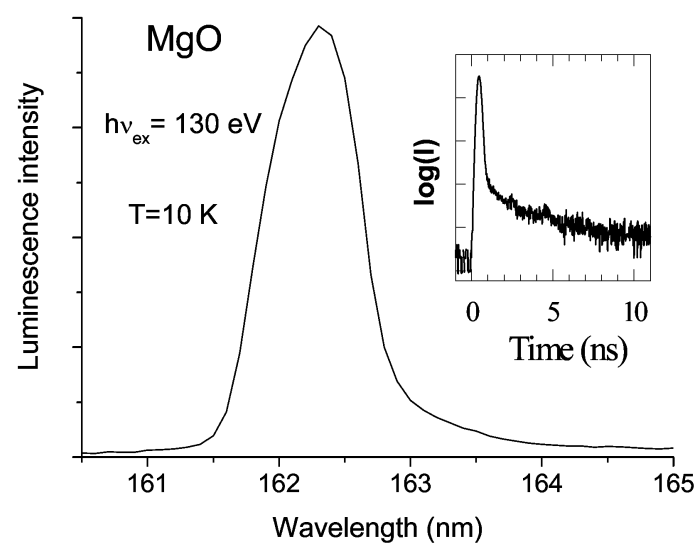

Fig. 4. Luminescence spectra of $\mathrm{MgO}$ single crystal measured under excitation by $130 \mathrm{eV}$ photons at $10 \mathrm{~K}$. The bandwidth is determined here by the spectral resolution of the monochromator which is $\sim 1 \mathrm{~nm}$. Inset shows decay of edge luminescence of excitons (Log scale is used).

STE luminescence decreases also at the $\mathrm{F}^{-} 1 s$ edge, whereas that of CL increases in this spectral region. Such a behavior of the excitation spectrum of CL near $F^{-} 1 s$ absorption edges has been reported earlier for a $\mathrm{BaF}_{2}$ crystal [14], [15].

A peculiar behavior of the excitation spectrum for CL in a $\mathrm{KMgF}_{3}$ crystal at the $\mathrm{F}^{-} 1 s$ edge can be interpreted similar to a $\mathrm{BaF}_{2}$ crystal [15] as an inter-atomic (F-K) cross-relaxation process of holes. The increase of CL intensity above the $\mathrm{F}^{-} 1 s$ edge means that the Auger decay of the $\mathrm{F}^{-} 1 s$ core hole finally creates the $K^{+} 3 p$ shell holes needed for CL. The mechanism behind the process was proposed in [16], which explains the effect by resonant electron-transfer relaxation between the outer $n p$ level of the metal ion and the $2 p$ level of fluorine ion studied for alkali metal and alkaline-earth fluorides using Auger spectroscopy. Among all alkali fluorides this effect is strongest just for potassium fluoride.

\section{B. Edge Luminescence of Excitons From $\mathrm{MgO}$}

Pure $\mathrm{MgO}$ emits narrow-band luminescence at $162 \mathrm{~nm}$ (Fig. 4) under XUV excitation at low temperature. This emission is due to edge luminescence of excitons [4], [5] which is interpreted as luminescence of free or bound excitons of large radius in regular or defect-related sites of the crystal. Although the peak intensity of narrow-line excitonic emission from $\mathrm{MgO}$ is rather strong, the integrated emission intensity in comparison with lower energy luminescence bands (see [5] and references therein) is not very high. The decay time of the luminescence of large radius excitonic states of $\mathrm{MgO}$ is in picosecond range, i.e., very high time resolution can be obtained with this scintillator material. As depicted in the inset of Fig. 4, the dominating ultrafast component conforms to the instrumental response of the detection system. The edge luminescence of excitons is practically quenched at $T=120 \mathrm{~K}$. However, rather weak and thermally broadened structures in VUV can be detected in $\mathrm{MgO}$ at room temperature as well, but the efficiency of this emission is too small for any applied purpose.

Luminescence properties of $\mathrm{MgO}$ have already been investigated under excitation by XUV radiation covering the $\mathrm{Mg}$ $2 p$ and $O 1 s$ absorption edges [13]. The excitation spectrum of $\mathrm{MgO}$ excitonic luminescence shows a typical "inverted"

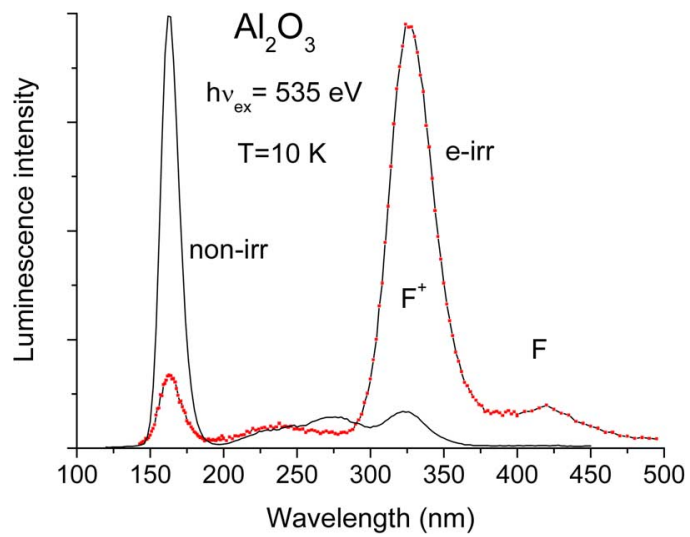

Fig. 5. Luminescence spectra of non-irradiated and electron-irradiated $\alpha-\mathrm{Al}_{2} \mathrm{O}_{3}$ single crystals measured under excitation by $535 \mathrm{eV}$ photons at $10 \mathrm{~K}$.

behavior with respect to the absorption spectrum at the $\mathrm{Mg}$ $2 p$ edge. Recently, $\mathrm{MgO}$ crystals have been studied near the $\mathrm{O} 1 s$ edge using X-ray fluorescence spectroscopy, resulting in high quality absorption spectra [17]. A detailed comparison (not shown) with that one confirms that the efficiency of edge exciton luminescence has a positive jump at the $\mathrm{O} 1 s$ edge, whereas other emissions exhibit normal behavior. This effect was interpreted in [13] as the result of an increased probability of valence exciton formation under excitation at the $\mathrm{O} 1 s$ edge, since the main decay channels of the $\mathrm{O} 1 s$ core hole create mainly valence holes, which thereafter tend to form excitons.

\section{Luminescence of Self-Shrunk Excitons From $\mathrm{Al}_{2} \mathrm{O}_{3}$}

Emission spectra of $\alpha-\mathrm{Al}_{2} \mathrm{O}_{3}$ single crystals under $535 \mathrm{eV}$ excitation at $10 \mathrm{~K}$ are shown in Fig. 5. The measurements were performed in particular for a crystal which was previously irradiated in air at room temperature by $50 \mathrm{MeV}$ electrons with a fluence of $6 \times 10^{17} \mathrm{e} / \mathrm{cm}^{2}$. The fast broadband VUV intrinsic emission at $166 \mathrm{~nm}$ dominates in the spectrum of non-irradiated $\alpha-\mathrm{Al}_{2} \mathrm{O}_{3}$. It is well known that this emission is effectively excited at the direct optical creation of excitons in the region $8.8-9.3 \mathrm{eV}$ or at the recombination of free electrons and free holes under high-density excitation by electron beam [6], [7]. Since no evidence of self-trapping for both electrons and holes has been obtained for $\mathrm{Al}_{2} \mathrm{O}_{3}$, the $166 \mathrm{~nm}$ emission was ascribed to the radiative decay of self-shrunk excitons (see [6] and references therein). Such kind of immobile excitons can exist in a system where an electron and a hole do not separately undergo self-trapping, while the sum of their deformation potentials is sufficient for the recombination formation of a localized exciton. Various exciton formation mechanisms from free and self-trapped carriers (i.e., electron and holes) in the field of acoustic phonons have been theoretically analyzed in [18]. The studied crystal shows also luminescence of $\mathrm{F}^{+}(325 \mathrm{~nm})$ and $\mathrm{F}$ $(400 \mathrm{~nm})$ centers primarily resulting from electron irradiation.

The excitation spectra of both excitonic $(166 \mathrm{~nm})$ and $\mathrm{F}^{+}$center $(325 \mathrm{~nm})$ luminescence were measured in the energy range of the $\mathrm{Al} 2 p$-edge $(75-130 \mathrm{eV})$ and $\mathrm{O} 1 s$-edge $(535-575 \mathrm{eV})$. The obtained excitation spectra of those emissions (not shown) are similar to the spectra obtained in [13] for excitonic luminescence of (non-irradiated) $\alpha-\mathrm{Al}_{2} \mathrm{O}_{3}$ and its 


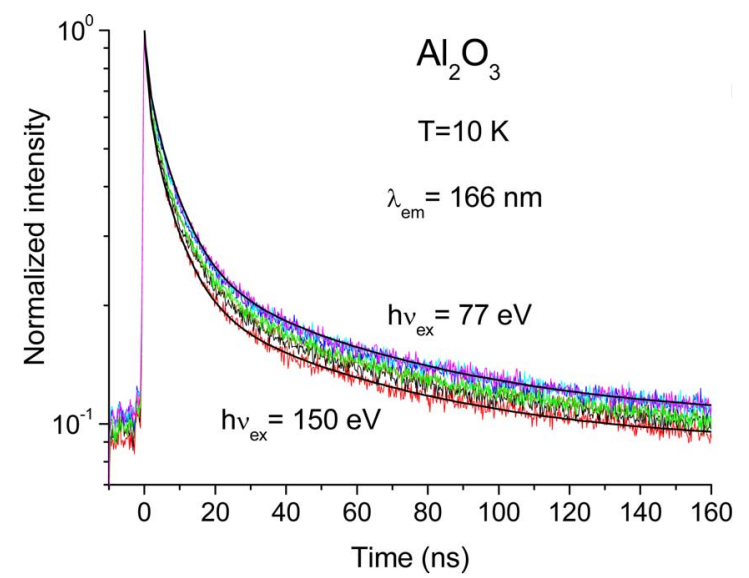

Fig. 6. Decay curves of excitonic luminescence (at $166 \mathrm{~nm}$ ) from $\mathrm{Al}_{2} \mathrm{O}_{3}$ measured at a temperature of $10 \mathrm{~K}$ under excitation by photons with different energies: 77, 79, 92, 100, 120, 126 and $150 \mathrm{eV}$. Solid curves are best fits with three-exponential decay for excitation energies 77 and $150 \mathrm{eV}$.

features are "inverted" with respect to absorption spectrum of $\alpha-\mathrm{Al}_{2} \mathrm{O}_{3}$ at both edges.

The decay curves of excitonic emission from $\alpha-\mathrm{Al}_{2} \mathrm{O}_{3}$ are presented in Fig. 6. No remarkable difference was found for decay curves measured from non-irradiated and electron-irradiated samples under excitation by photons with the same energies. The decay law is always non-exponential with a considerable contribution of the slow emission component. Remarkable changes of decay kinetics can be seen under excitation by photons of different energies. In particular, the initial stage of decay becomes faster for excitation by photons of higher energies. The decay curves can be well simulated by a three-exponential decay function, e.g., for excitation by $77 \mathrm{eV}$ with $\tau_{1}=1.06 \mathrm{~ns}$, $\tau_{2}=7.8 \mathrm{~ns}, \tau_{3}=56.3 \mathrm{~ns}$ and for excitation by $150 \mathrm{eV}$ with $\tau_{1}=1.05 \mathrm{~ns}, \tau_{2}=7.1 \mathrm{~ns}, \tau_{3}=53.7 \mathrm{~ns}$ (see Fig. 6). These features indicate a rather complicated mechanism of creation of self-shrunk excitons under XUV excitation, as well as the presence of some quenching of excitonic luminescence for excitation by high-energy photons. For comparison, in highly pure Czochralski grown $\alpha-\mathrm{Al}_{2} \mathrm{O}_{3}$ the decay of the $7.6 \mathrm{eV}$ intrinsic excitonic emission observed after excitation with $8.98 \mathrm{eV}$ photons has a lifetime of $7 \mathrm{~ns}$. The luminescence decay is well described by a single exponential [19].

\section{D. $5 d-4 f$ Luminescence of $G d^{3+}$ Ions in Fluoride Matrices}

Recently it has been found that several $\mathrm{Gd}^{3+}$ containing wide band-gap fluoride crystals possess fast luminescence in the VUV spectral range due to interconfigurational $5 d-4 f$ transitions in $\mathrm{Gd}^{3+}$ [10]-[12]. It is well known that some trivalent rare earth (RE) ions show radiative decay from their mixed $4 f^{\mathrm{n}-1} 5 d$ electronic configuration, i.e., such RE ions emit so called $5 d-4 f$ luminescence. This luminescence is caused by transitions from the lowest level of $4 f^{n-1} 5 d$ electronic configuration ( $5 d$ level) to the ground state or some excited levels of $4 f^{\mathrm{n}}$ electronic configuration of the $\mathrm{RE}^{3+}$ ion. For the first half of the lanthanide series, transitions from the lowest $5 d$ level into the $4 f$ levels are spin-allowed and $5 d-4 f$ luminescence is fast with typical lifetimes in the nanosecond range. In the

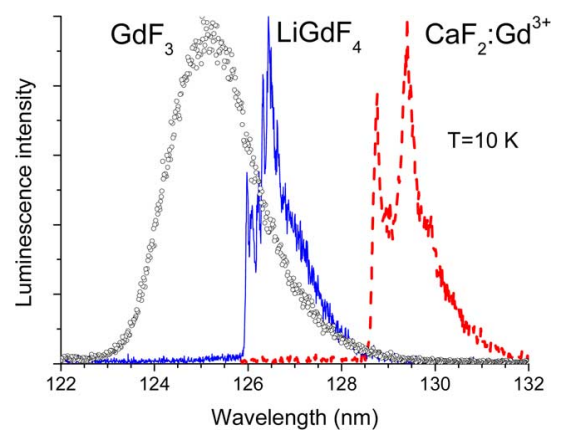

Fig. 7. VUV luminescence spectra of three $\mathrm{Gd}^{3+}$ containing compounds measured at $10 \mathrm{~K}$. Spectra were recorded under VUV excitation with a spectral resolution of $0.05 \mathrm{~nm}[10]-[12]$.

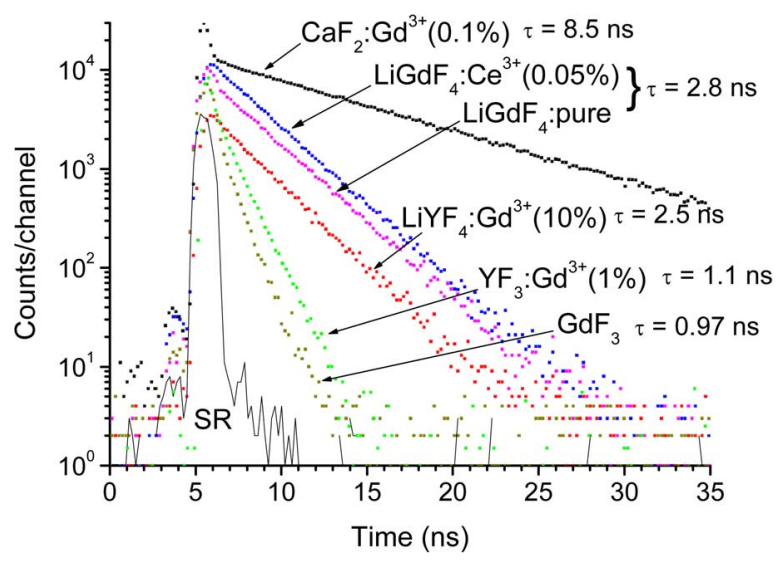

Fig. 8. Decay curves of VUV luminescence from different $\mathrm{Gd}^{3+}$ containing compounds measured under direct $4 f-5 d$ excitation. The respective decay times are indicated in the Figure. The time profile of SR excitation pulse (including instrumental response) is also shown.

second half of series, transitions from the lowest $5 d$ state are spin-forbidden, and such $5 d-4 f$ luminescence is slow with lifetimes in the $\mu$ s range.

Examples of VUV emission spectra due to $5 d-4 f$ transitions in $\mathrm{Gd}^{3+}$ are shown in Fig. 7. The spectra from the $\mathrm{LiGdF}_{4}$ and $\mathrm{CaF}_{2}: \mathrm{Gd}^{3+}$ crystals have maxima at $\sim 126.5$ and $129.5 \mathrm{~nm}$ respectively and show well-pronounced fine structure due to zerophonon lines and vibronics. The spectrum from $\mathrm{LiYF}_{4}: \mathrm{Gd}^{3+}$ (not shown here) has nearly the same shape as that from the $\mathrm{LiGdF}_{4}$ crystal but is slightly shifted to longer wavelengths. The VUV emission spectrum of $\mathrm{GdF}_{3}$ is situated at shorter wavelengths than all other studied crystals and shows a single structureless band centered at $\sim 125 \mathrm{~nm}$. A similar emission spectrum is observed from $\mathrm{YF}_{3}: \mathrm{Gd}^{3+}$ (not shown). The decay times of VUV luminescence from all $\mathrm{Gd}^{3+}$-containing samples are in the nanosecond range. Fig. 8 presents the survey of data from [10]-[12] on $\mathrm{Gd}^{3+} 5 d-4 f$ luminescence decay under VUV excitation in different $\mathrm{Gd}^{3+}$-containing compounds. At $T \sim 10 \mathrm{~K}$, the following decay times were obtained: $8.5 \mathrm{~ns}$ for $\mathrm{CaF}_{2}: \mathrm{Gd}^{3+}, 2.8 \mathrm{~ns}$ for $\mathrm{LiGdF}_{4}, 2.5 \mathrm{~ns}$ for $\mathrm{LiYF}_{4}: \mathrm{Gd}^{3+}, 0.97$ ns for $\mathrm{GdF}_{3}$, and $1.1 \mathrm{~ns}$ for $\mathrm{YF}_{3}: \mathrm{Gd}^{3+}$.

VUV luminescence from all $\mathrm{Gd}^{3+}$ compounds is observed only at low temperatures. As an example, the temperature dependence of VUV luminescence from $\mathrm{LiYF}_{4}: \mathrm{Gd}^{3+}(10 \%)$ is shown in Fig. 9. The emission spectra were measured here with 


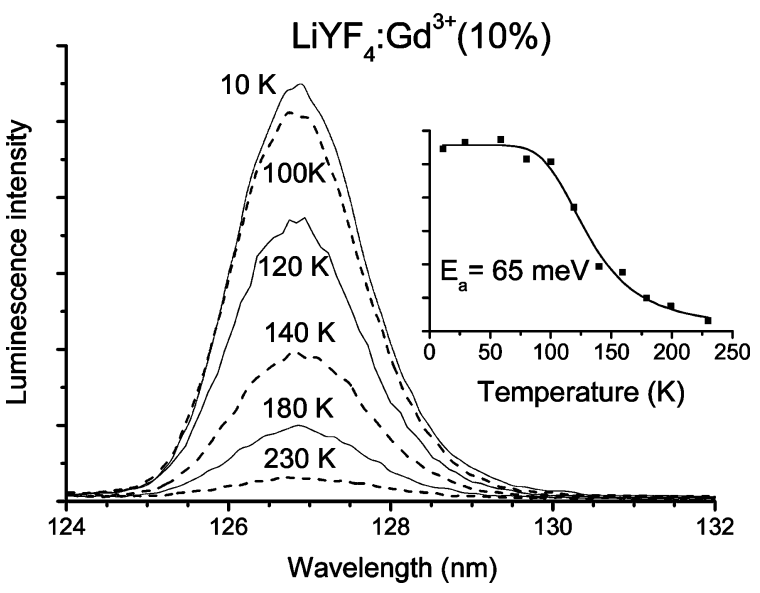

Fig. 9. Temperature dependence of the VUV luminescence from $\mathrm{LiYF}_{4}$ : $\mathrm{Gd}^{3+}(10 \%)$. In the inset, the integrated intensity of the emission is plotted as a function of temperature, together with the simulation curve obtained as the best fit with the Mott formula. $E_{a}$ is activation energy of thermal quenching.

a moderate spectral resolution in order to prevent possible radiation damage, and accordingly the fine structure in the spectra was not revealed. The dependence of integrated luminescence intensity on temperature was approximated by the Mott formula, $I(T) / I(0)=\left(1+A \exp \left(-E_{a} / k_{B} T\right)\right)^{-1}$ (shown in the inset of Fig. 9). An activation energy $E_{a} \sim 65 \mathrm{meV}$ for thermal quenching was obtained from the fit. Around $250 \mathrm{~K}$, the VUV luminescence is quenched completely. Thermal quenching of $\mathrm{Gd}^{3+} 5 d-4 f$ luminescence is attributed to phonon assisted nonradiative relaxation from the lowest $\mathrm{Gd}^{3+} 5 d$ level to lower-lying $4 f$ levels (many $4 f$ levels are located in the same energy region as the $5 d$ state). The direct $5 d-4 f$ non-radiative transitions (at low temperature) are heavily spin-forbidden [10]-[12].

The possible dependence of decay properties of VUV luminescence from $\mathrm{Gd}^{3+}$ containing materials upon photon energy of irradiation has been analyzed under excitation of the samples around the $\mathrm{Gd}^{3+} 4 d$ absorption edge (see Fig. 10). The absorption coefficient is strongly increased in this region (known as "giant resonance" [20]), resulting in smaller thickness of the layer in which radiation is absorbed and accordingly to stronger near-surface losses. Indeed, excitation and absorption spectra show "inverted" behavior in this region. However, no remarkable changes of decay curves (shown in the inset of Fig. 10) have been observed for different excitation photon energies although some shortening of decay (the appearance of the faster initial stage of decay) is observed under XUV excitation compared to direct $4 f-5 d$ excitation by VUV radiation.

One more type of VUV emitters exists which is based on $5 d-$ $4 f$ luminescence of $\mathrm{Lu}^{3+}$ ion incorporated into wide band-gap fluoride matrices [10], [11]. The wavelengths of these emissions are even shorter $(124 \mathrm{~nm})$ than those of $\mathrm{Gd}^{3+}$ based emitters. However, the decay for such kind of luminescence is slow because it is due to spin-forbidden $5 d-4 f$ transitions.

\section{E. General Consideration}

The main characteristics of VUV luminescence from the studied crystals are listed in Table I. The estimation of the relative light outputs of different scintillator materials under

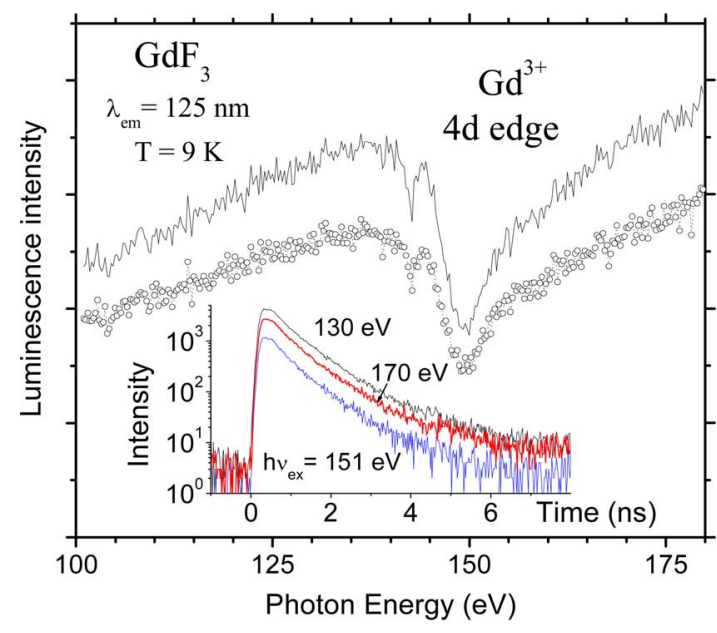

Fig. 10. Excitation spectra of fast (circles, $\Delta=1.75 \mathrm{~ns}, \delta=0.37 \mathrm{~ns}$ ) and time-integrated (solid curve) VUV luminescence from $\mathrm{GdF}_{3}$ crystal at $9 \mathrm{~K}$ in the range of the Gd $4 d$ absorption. The inset shows decay curves of VUV luminescence measured under excitation by photons of different energies.

TABLE I

MAIN PROPERTIES OF DIFFERENT VUV EMITTERS

\begin{tabular}{lcc}
\hline Crystal & $\begin{array}{c}\text { Emission wavelength } \\
(\mathrm{nm})\end{array}$ & Decay time (ns) \\
\hline $\mathrm{KMgF}_{3}$ & 150,170 & $\sim 1.5$ \\
$\mathrm{MgO}$ & 162 & $<0.2$ \\
$\mathrm{Al}_{2} \mathrm{O}_{3}$ & 166 & $\sim 7$ \\
$\mathrm{LiGdF}_{4}$ & 126.5 & 2.8 \\
$\mathrm{LiYF}_{4}: \mathrm{Gd}$ & 127 & 2.5 \\
$\mathrm{GdF}_{3}$ & 125 & 1 \\
\hline
\end{tabular}

XUV excitation is not an easy task, since the studied scintillators show narrow emission lines as well as broadband luminescence with very different efficiencies. Therefore luminescence spectra were recorded using different settings (slit widths) in the excitation as well as in the detection channel, which introduces uncertainties in quantitative comparison of light yields of studied materials. The integrated intensity of broadband emissions $\left(\mathrm{Al}_{2} \mathrm{O}_{3}, \mathrm{KMgF}_{4}\right)$ is higher than that of edge emission observed from $\mathrm{MgO}$. The d-f emissions in $\mathrm{LiGdF}_{4}$ and $\mathrm{GdF}_{3}$, however, produce less light compared to VUV luminescence of pure MgO. In addition, the selection of excitation energy plays a significant role because the location of inner-shell edges strongly influences absorption as well as relaxation processes in the scintillators. Nevertheless, taking into account above-mentioned limitations, such investigations provide useful information about relative light yields, since each XUV photon creates many electron-hole pairs in the crystal, similar to the processes of energy dissipation in the scintillator under irradiation by high-energy photons or particles.

The specific spectral and temporal behavior of VUV luminescence in different crystals is due to the features of energy transfer mechanisms for each kind of luminescence: CL, different kinds of excitonic emission, $\mathrm{d}-\mathrm{f}$ luminescence of rare earth ions. In most scintillating crystals, the so-called recombination mechanism is responsible for the energy transfer from the host crystal to luminescence centers, which are usually some impurity ions. This mechanism suggests that one carrier (e.g., a hole) is first captured by the impurity ion and then the partner 
(electron) recombines with this hole resulting in excitation of the ion to some excited level. Both processes are fast and do not lead to any increase of luminescence decay time. However, the processes of carrier trapping by some other traps (defect centers), in particular hole self-trapping, can compete with the capture of carriers by the luminescence centers. In this case some delay of the energy transfer to luminescence centers can occur resulting in slower decay of luminescence, sometimes with an initial rising part in the luminescence timing profile with a duration corresponding to characteristic time of the energy transfer process.

In the case of "conventional" scintillators based on luminescence of some impurity ions excited via the recombination mechanism, it is usually assumed that practically all electrons and holes created after the absorption of high-energy photon with energy $E \gamma$ will recombine eventually at the luminescence centers. If the intrinsic luminescence quantum efficiency of the luminescence centers is unity, the light yield of the scintillator $\eta$ will be determined by the number of created electron-hole pairs $n_{e^{-} h}$. This can be expressed as: $\eta \sim n_{e^{-} h}=E \gamma / \beta \cdot E_{g}$, where $\beta$ is the conversion efficiency for creating electron-hole pairs [21]-[23]. As was found both from experiments and theory, $\beta$ is about $2-3$ times higher than the band-gap energy $E_{g}$. However, it seems that the recombination mechanism and accordingly the above estimation of the light yield are not applicable to all crystals studied here, in particular because of rather high energies of the emitted photons.

A good approximation for the energy that can be transferred from the host to the luminescence center by the recombination mechanism is the photon energy of STE emission (if it exists). This value also limits the maximum energy of photons that can be emitted from the luminescence center. In all such crystals there is a rather large Stokes shift (several eV) between the energy of direct optical creation of (free) excitons (which is less by few tenths of $\mathrm{eV}$ than the value of the band-gap $E_{g}$ ) and the photon energy of STE emission. Accordingly, one cannot expect that the recombination mechanism works if the energy of emitted photons is close to the value of the band-gap.

The mechanism of the energy transfer for CL is obviously different and rather specific. CL is observed only if the hole is created in the (uppermost) core band. This leads to quite a moderate light yield in any CL scintillator ( $~ 1500$ photons/MeV) since it is necessary to "spend" considerably more energy for the creation of one photon of luminescence than for the case of creation of the hole in the valence band. However, the main factors determining the low light yield of CL scintillators are fast electron thermalization and core hole Auger decay [24]. On the other hand, no delay of the energy transfer is possible for CL since the core hole disappears in any case after its creation either radiatively via CL or as a result of Auger decay. Therefore, scintillation will be always fast for CL scintillators. Some weak dependence on excitation energy of CL decay time is due to different quenching mechanisms of CL [3], which are rather weak for such high-penetration radiation as $\gamma$-rays. A detailed analysis of CL quenching mechanisms in $\mathrm{BaF}_{2}$ under XUV excitation has been carried out in [25].

No self-trapping of excitons, electrons or holes has been detected in $\mathrm{MgO}$ crystals, i.e., no other types of intrinsic lumines- cence are observed from pure $\mathrm{MgO}$ except for the narrow edge emission due to large radius excitons. Doping with iso-electronic $\mathrm{Ca}^{2+}$ and $\mathrm{Be}^{2+}$ ions introduces small radius states, which emit broadband luminescence with maxima at 7.0 and $6.3 \mathrm{eV}$, respectively [5]. Latter emissions have higher integrated intensity, but their decay time is considerably longer (in ns range) and the achievable time-resolution is not so good as for pure $\mathrm{MgO}$ scintillator.

The $166 \mathrm{~nm}$ emission from $\mathrm{Al}_{2} \mathrm{O}_{3}$ is due to luminescence of so-called self-shrunk excitons. This kind of immobile excitons can exist in a system where an electron and a hole do not separately undergo self-trapping while the sum of their deformation potentials is sufficient for the recombination formation of a localized exciton [18]. Under high-energy excitation, the prevailing mechanism of formation of such excitons is the recombination of free electron-hole pairs, which is not efficient for band-to-band excitation by low-energy photons creating single electron-hole pairs. However, under XUV excitation the local density of electron-hole pairs created after the absorption of each high-energy photon is strongly increased and the probability of electron-hole recombination becomes much higher, resulting in rather high light yield of excitonic luminescence. The process of electron-hole recombination can be delayed by the presence of existing defects/traps. As a result, the decay becomes non-exponential with considerable fraction of the slow emission component.

An interesting comparison can be made with the luminescence characteristics of free and self-trapped excitons in rare gas solids (RGS) under XUV excitation [26]. Due to the lack of optical phonons in these mono-atomic crystals, energy relaxation of conduction band electrons occurs on a nanosecond timescale. This allows the distinction between prompt exciton luminescence from primary excitons that are created directly during the excitation, and delayed secondary excitons from electron-hole recombination processes. Under XUV excitation, conduction electrons with high kinetic energies are created, which can create additional prompt secondary excitons, if their kinetic energy exceeds the energy of a free exciton [27]. The prompt creation of excitons occurs through an electron-electron scattering process, which is essentially the same impact mechanism as discussed below in more detail for excitation of $5 \mathrm{~d}$ states of rare earth ions. The thresholds for this process can be clearly observed in time-resolved excitation spectra for the exciton luminescence in RGS in the region of valence and core excitations as summarized and analyzed in [28].

The mechanism of the energy transfer from the host crystal to $\mathrm{Gd}^{3+} 5 d-4 f$ luminescence cannot be the recombinational one since too much energy ( $\sim 10 \mathrm{eV}$, i.e., close to the band-gap value) should be transferred to the $\mathrm{Gd}^{3+}$ ion in order to excite it to the $5 d$ level. This fact is confirmed by measurements of $\mathrm{Gd}^{3+} 5 d-4 f$ luminescence excitation spectra in the region $h \nu>12 \mathrm{eV}$, corresponding to band-to-band transitions of the host crystals, where no $\mathrm{Gd}^{3+} 5 d-4 f$ luminescence was detected [10]-[12]. The dominant mechanism of the energy transfer in these systems is so-called impact mechanism, when fast photoelectrons excite directly (by impact) the $\mathrm{Gd}^{3+}$ ions to the $5 d$ level. For this process, the kinetic energy of such electrons must exceed the energy of $4 f-5 d$ excitation $E_{f d}$, i.e., the photon en- 
ergy of exciting radiation needs to be above the value $E_{g}+$ $E_{f d}$ which is $\sim 22 \mathrm{eV}$ for $\mathrm{LiGdF}_{4}$ and $\mathrm{GdF}_{3}$. The presence of this mechanism can be clearly seen in excitation spectra of $\mathrm{Gd}^{3+} 5 d-4 f$ luminescence measured in this region, where the threshold is observed for the $\mathrm{Gd}^{3+}$ luminescence excitation at photon energies $h \nu \sim 22 \mathrm{eV}[29]$. The same impact mechanism has been demonstrated to occur for excitation of $\mathrm{Er}^{3+} 5 d-4 f$ luminescence in fluoride crystals [30]. A comprehensive study of impact mechanism by methods of photoelectron and luminescence spectroscopy has been performed for $\mathrm{Tl}^{+}$impurity ions in alkali chlorides [31]. The efficiency of the impact mechanism of the energy transfer cannot be very high. However, for compounds with high concentration of $\mathrm{Gd}^{3+}$ ions, in particular for stoichiometric compounds, one can expect to obtain a light yield of the same order as for the well-known UV scintillator $\mathrm{CeF}_{3}$, in which the same impact mechanism is responsible for the scintillation emission due to $5 d-4 f$ transitions of $\mathrm{Ce}^{3+}$ [32], [33]. Taking into account that the photon energy of $\mathrm{Gd}^{3+}$ VUV luminescence is roughly 2.5 times larger than the photon energy of $\mathrm{CeF}_{3}$ emission, one can expect a light yield of stoichiometric $\mathrm{Gd}^{3+}$ compounds up to $\sim 1600$ photons/MeV. However our measurements have shown that the real light yield is much lower. The reason of this is most probably due to the fact that the efficiency of the impact mechanism decreases with the increase of the energy, which should be transferred to the excited ion by fast photoelectron.

The inverted behavior of the absorption spectrum and excitation spectrum of $\mathrm{Gd}^{3+} 5 d-4 f$ luminescence can be explained by near-surface losses of (fast) photoelectrons. However, if the electron has excited (by impact) the $\mathrm{Gd}^{3+}$ ion into the $5 d$ state, the energy will not be lost by the $\mathrm{Gd}^{3+}$ ion because of the localized type and short lifetime of the $5 d$ excitation. Accordingly, the decay time of $\mathrm{Gd}^{3+} 5 d-4 f$ luminescence under XUV excitation will be roughly the same as under $4 f-5 d$ excitation. However, some shortening of decay can appear due to quenching caused by interaction of neighbor excitations, the local density of which can be rather high under XUV excitation.

\section{CONCLUSION}

Inorganic materials offer a variety of VUV scintillators, which have very different spectral and temporal properties. The $\mathrm{Gd}^{3+}$-containing fluoride compounds show the shortest-wavelength emission, which overlaps spectrally with emission of liquid argon, but they have the lowest light yield among all studied VUV emitters. $\mathrm{Al}_{2} \mathrm{O}_{3}$ has a much higher light yield of VUV emission, but timing properties of this emission are not optimal, with a considerable contribution of the slow emission component. $\mathrm{MgO}$ excitonic emission has very fast decay and a high-intense narrow-line spectrum but its spectrally integrated emission intensity is not as high as that of broad-band emission from CL scintillators. CL crystals (KF-based) can be considered as the best VUV scintillator among those studied in this work, with relatively high light yield and fast $(\sim 1 \mathrm{~ns})$ decay, but its emission spectrum does not fit well the spectral range of emission from liquid xenon and argon scintillators. The general recommendation is that according to the specific need of the particular application, VUV emitters with the required performance have to be selected.

\section{ACKNOWLEDGMENT}

The authors would like to thank G. Stryganyuk for his assistance in measurements with VUV synchrotron radiation, and A. Kikas and G. Zimmerer for valuable discussions.

\section{REFERENCES}

[1] R. Gaitskell, "Noble travails: Noble liquid dark matter detectors," 2007 [Online]. Available: http://xenon.astro.columbia.edu/talks/APS2007.

[2] V. N. Makhov and N. M. Khaidukov, "Cross-luminescence peculiarities of complex KF-based fluorides," Nucl. Instrum. Meth. A, vol. 308, no. 1-2, pp. 205-207, 1991.

[3] I. A. Kamenskikh, M. A. MacDonald, V. N. Makhov, V. V. Mikhailin, I. H. Munro, and M. A. Terekhin, "Fast crystalline scintillators for high counting rate X-ray detectors," Nucl. Instrum. Meth. A, vol. 348, no. 1-2, pp. 542-545, 1994.

[4] E. Feldbach, I. Kuusmann, and G. Zimmerer, "Excitons and edge luminescence of MgO," J. Lumin., vol. 24/25, pp. 433-436, 1981.

[5] M. Kirm, A. Lushchik, and C. Lushchik, "Creation of groups of spatially correlated excitations in wide-gap solids," Phys. Stat. Sol. (a), vol. 202, no. 2, pp. 213-220, 2005.

[6] M. Kirm, G. Zimmerer, E. Feldbach, A. Lushchik, C. Lushchik, and F. Savikhin, "Self-trapping and multiplication of electronic excitations in $\mathrm{Al}_{2} \mathrm{O}_{3}$ and $\mathrm{Al}_{2} \mathrm{O}_{3}$ : Sc crystals," Phys. Rev. B, vol. 60, no. 1, pp. 502-510, 1999.

[7] M. Kirm, V. V. Harutunyan, V. N. Makhov, and S. Vielhauer, "VUV luminescence of as-grown and electron irradiated corundum single crystals," Proc. SPIE, vol. 5946, pp. 41-46, 2005.

[8] V. N. Makhov, J. Y. Gesland, N. M. Khaidukov, N. Y. Kirikova, M. Kirm, J. C. Krupa, M. Queffelec, T. V. Ouvarova, and G. Zimmerer, "VUV scintillators based on d-f transitions in rare earth ions," in Proc. Fifth Int. Conf. Inorganic Scintillators and Their Applications, Moscow, 1999, pp. 369-374.

[9] V. N. Makhov, N. Y. Kirikova, M. Kirm, J. C. Krupa, P. Liblik, A Lushchik, C. Lushchik, E. Negodin, and G. Zimmerer, "Luminescence properties of $\mathrm{YPO}_{4}: \mathrm{Nd}^{3+}$ : A promising VUV scintillator material," Nucl. Instrum. Meth. A, vol. 486, no. 1-2, pp. 437-442, 2002.

[10] M. Kirm, J. C. Krupa, V. N. Makhov, M. True, S. Vielhauer, and G. Zimmerer, "High resolution vacuum ultraviolet spectroscopy of $5 d-$ $4 f$ transitions in Gd and Lu fluorides," Phys. Rev. B, vol. 70, no. 24, pp. 241101(R)-241101(R), 2004.

[11] M. Kirm, G. Stryganyuk, S. Vielhauer, G. Zimmerer, V. N. Makhov, B. Z. Malkin, O. V. Solovyev, R. Y. Abdulsabirov, and S. L. Korableva, "Vacuum ultraviolet $5 d-4 f$ luminescence of $\mathrm{Gd}^{3+}$ and $\mathrm{Lu}^{3+}$ ions in fluoride matrices," Phys. Rev. B, vol. 75, no. 7, p. 075111, 2007.

[12] V. N. Makhov, S. K. Batygov, L. N. Dmitruk, M. Kirm, G. Stryganyuk, and G. Zimmerer, "VUV $5 d-4 f$ luminescence of $\mathrm{Gd}^{3+}$ doped into $\mathrm{Caf}_{2}$, ," Phys. Stat. Solidi (c), vol. 4, no. 3, pp. 881-884, 2007.

[13] M. Kirm, A. Lushchik, C. Lushchik, and S. V. Zimmerer, "Luminescence of pure and doped $\mathrm{Al}_{2} \mathrm{O}_{3}$ and $\mathrm{MgO}$ single crystals under innershell excitation," J. Lumin., vol. 102-103, pp. 307-312, 2003.

[14] J. Voss, "The scanning soft X-ray microscope at Hasylab: Imaging and spectroscopy of photoelectrons, photoluminescence, desorbed ions, reflected, scattered and transmitted light," J. Electron Spectrosc. Relat. Phenom., vol. 84, no. 1-3, pp. 29-44, 1997.

[15] M. Kirm, A. Lushchik, C. Lushchik, V. N. Makhov, E. Negodin, S Vielhauer, and G. Zimmerer, "VUV luminescence of $\mathrm{BaF}_{2}, \mathrm{BaF}_{2}$ : $\mathrm{Nd}$ and $\mathrm{BaY}_{2} \mathrm{~F}_{8}$ crystals under inner-shell excitation," Nucl. Instrum. Meth. A, vol. 486, no. 1-2, pp. 422-425, 2002.

[16] O. Benka and M. Uda, "Resonant electron-transfer relaxation observed in fluorine K Auger spectra," Phys. Rev. Lett., vol. 56, no. 16, pp. 1667-1670, 1986.

[17] A. Kikas, T. Käämbre, V Kisand, A. Saar, K. Kooser, E. Nõmmiste, and I. Martsinson, "Resonant inelastic X-ray scattering at the K edge of oxygen and fluorine in insulators," J. Electron Spectrosc. Relat. Phenom., vol. 144-147, pp. 845-848, 2005.

[18] A. Sumi, "Phase diagram of an exciton in the phonon field," J. Phys. Soc. Jpn., vol. 43, no. 4, pp. 1286-1294, 1977.

[19] A. Lushchik, E. Feldbach, M. Kirm, P. Liblik, C. Lushchik, I. Martinson, F. Savikhin, and G. Zimmerer, "Spectral-kinetic study of self-trapping and multiplication of electronic excitations in $\mathrm{Al}_{2} \mathrm{O}_{3}$ crystals," J. Electron Spectrosc. Relat. Phenom., vol. 101-103, pp. 587-591, 1999

[20] T. M. Zimkina, V. A. Fomichev, S. A. Gribovsky, and I. I. Zhukova, "Some peculiarities in X-ray absorption by lanthanium group rare earth metals," Sov. Phys._Solid State, vol. 9, no. 5, pp. 1128-1131, 1967. 
[21] D. J. Robbins, "On predicting the maximum efficiency of phosphor systems excited by ionizing-radiation," J. Electrochem. Soc., vol. 127, pp. 2694-2702, 1980

[22] A. Lempicki, A. J. Wojtowicz, and E. Berman, "Fundamental limits of scintillator performance," Nucl. Instrum. Meth. A, vol. 333, pp. 304-311, 1993.

[23] P. A. Rodnyi, P. Dorenbos, and C. W. E. van Eijk, "Energy loss in inorganic scintillators," Phys. Stat. Sol. (b), vol. 187, pp. 15-29, 1995.

[24] A. N. Vasil'ev, "Elementary processes in scintillation and their interconnection in scintillation process," in Proc. 8th Int. Conf. on Inorganic Scintillators and Their Use in Scientific and Industrial Applications, Alushta, Ukraine, 2005, pp. 1-6.

[25] M. A. Terekhin, A. N. Vasil'ev, M. Kamada, E. Nakamura, and S. Kubota, "Effect of quenching processes on the decay of fast luminescence from barium fluoride excited by VUV synchrotron radiation," Phys. Rev. B, vol. 52, no. 5, pp. 3117-3121, 1995.

[26] S. Vielhauer, M. Kirm, V. Kisand, E. Negodin, E. Sombrowski, B. Steeg, and G. Zimmerer, "Excitonic side bands of inner-shell excitation in rare gas solids," Surf. Rev. Lett., vol. 9, no. 2, pp. 1333-1338, 2002.

[27] S. Vielhauer, "Innerschalenanregungen und sekundäre exzitonen in edelgasfestkörpern," Ph.D. dissertation, Univ. of Hamburg, Hamburg, 2003.
[28] M. Kirm, V. Kisand, E. Sombrowski, B. Steeg, S. Vielhauer, and G. Zimmerer, "Prompt and delayed secondary excitons in rare-gas solids," Fizika Nizkikh Temperatur, vol. 29, no. 9-10, pp. 1081-1092, 2003.

[29] M. Kirm, V. N. Makhov, and S. Vielhauer, unpublished results.

[30] V.N. Makhov, N.M. Khaidukov, N.Y. Kirikova, M. Kirm, J.C. Krupa, T.V. Ouvarova, and G. Zimmerer, "VUV emission of rare earth ions doped into fluoride crystals," J. Lumin., vol. 87-89, pp. 1005-1007, 2000.

[31] E. Feldbach, M. Kamada, M. Kirm, A. Lushchik, C. Lushchik, and I. Martinson, "Direct excitation of $\mathrm{Tl}^{+}$impurity ions by hot photoelectrons in wide-gap crystals," Phys. Rev. B, vol. 56, no. 21, pp. 13908-13915, 1997.

[32] Y. M. Aleksandrov, V. N. Makhov, and M. N. Yakimenko, "Impact excitation of impurity centers in $\mathrm{LaF}_{3}$ crystals activated with rare-earth elements," Sov. Phys._Solid State, vol. 29, no. 6, pp. 1092-1093, 1987.

[33] C. Pedrini, B. Moine, D. Bouttet, A. N. Belsky, V. V. Mikhailin, A. N. Vasil'ev, and E. I. Zinin, "Time-resolved luminescence of $\mathrm{CeF}_{3}$ crystals excited by X-ray synchrotron radiation," Chem. Phys. Lett., vol. 206, no. 5-6, pp. 470-474, 1993. 